\title{
Independent motion induction in separated portions of the visual field
}

\author{
WALTER C. GOGEL \\ University of California, Santa Barbara, California 93106
}

\begin{abstract}
As an example of induced motion, a stationary disk enclosed by a moving frame appears to move in a direction opposite to the physical motion of the frame. In the present study, either one moving frame with an enclosed stationary disk (a single frame-disk stimulus) was presented or two frame-disk stimuli were visible simultaneously in separated portions of the visual field. With the two frame-disk stimuli, the two frames moved repetitively right and left in opposite phase. Reports of motion from the two frame-disk stimuli indicate that induced motion in opposite directions can occur in separated portions of the visual field. The results suggest that not all instances of induced motion can be explained in terms of the tendency to displace the perceptual straight-ahead toward the center of the visual field (the Roelofs effect).
\end{abstract}

Induced motion often is illustrated by the situation in which a frame, physically moving right and left, surrounds a physically stationary disk with both the frame and the disk in the same frontoparallel plane. If no objects except the frame and disk are visible, it usually is found that the disk will appear to move in a direction (phase) opposite to that of the physical motion of the frame (Duncker, 1929). A plausible explanation of induced motion has been proposed by Brosgole (1966) in terms of the Roelofs effect. Roelofs (1935) has shown that a visual stimulus asymmetrically located with respect to the observer's straight-ahead direction will cause the apparent straight-ahead to deviate toward the physical center of the stimulus. Moving a frame right and left, therefore, will cause the physical correlate of the apparent straight-ahead to move with the frame. It follows that a physically stationary object located within the frame will systematically change its perceived direction relative to the perceived straight-ahead, and, as a result, it will appear to move with a phase opposite to that of the frame.

The explanation in terms of the Roelofs effect, despite its plausibility, does not seem to be appropriate to the full range of phenomena associated with induced motion. Sugarman and Cohen (1968) have concluded that the Roelofs effect cannot account for the full amount of induced displacement which occurs in a test object as a result of simultaneously displacing and moving the induction field. Gogel and Koslow $(1971,1972)$ found that the effectiveness of an induction object in producing an apparent motion in a test object decreased as the test and induction objects were increasingly separated in apparent depth without an appreciable change

This investigation was supported by U.S. Public Health Service Grant MH 15651 from the National Institute of Mental Health. The author wishes to thank Robert Newton for his assistance in the collection and analysis of the data. in apparent direction. It is also evident that the Roelofs effect cannot explain induced motions that occur in opposite directions in different parts of the same visual field. One example of such opposite induced motions was described by Duncker (1929). He found that the physical rotation of a large disk could induce an opposite apparent rotation in a smaller, physically stationary disk, with the latter disk directionally centered on the large disk. The induced motion at the opposite ends of any diameter of the smaller disk was opposite in direction. Duncker suggested that this result was opposed to an explanation of induction in terms of eye movements. It also cannot be explained in terms of the Roelofs effect.

Although Brosgole's (1966) theory of induced motion does not explain the induction of apparent rotary motion, it might be essential for explaining the process involved in linear motion induction that occurs between objects in the same frontoparallel plane. The purpose of the present study was to determine whether an induction of apparent linear motions of opposite phase can occur simultaneously in different portions of the same frontoparallel plane. If such opposite inductions occur, it is unlikely that they are the result of changes in the physical correlate of the perceived straight-ahead.

\section{METHOD}

\section{Apparatus}

The experimental conditions are illustrated by the perspective drawing of Figure 1. Two frame-disk stimuli were presented simultaneously, with both stimuli at either a near $(91.4 \mathrm{~cm})$ or a far $(182.8 \mathrm{~cm})$ distance from the observer. As indicated by the arrows in Figure 1 , as one frame moved laterally in one direction, the other frame moved laterally in the other direction, that is, the phase of motion of the two frames was opposite. If induced motion were to occur in the physically stationary disks under these conditions, each disk would appear to move with a phase opposite to that of its surrounding frame and the 


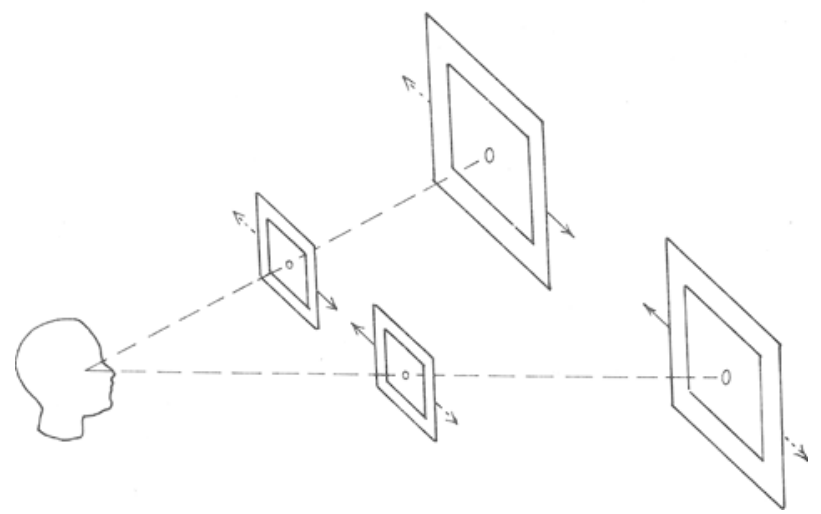

Figure 1. Schematic drawing of the presentation of either a pair of near frames and disks or a pair of far frames and disks. The disks were physically stationary and, as is indicated by the arrows, the frames of a pair physically moved in a phase opposite to each other.

two disks would appear to move opposite in phase to each other. The frames were formed from electroluminescent strips and were moved laterally by a gear and chain drive attached to an instantly reversible electric motor. At the near distance, the electroluminescent strips were $1.3 \mathrm{~cm}$ wide, the frames were $8.9 \mathrm{~cm}$ on a side, the disks were $.27 \mathrm{~cm}$ in diam, and each frame moved $1.9 \mathrm{~cm}$ from left to right at a speed of $1.34 \mathrm{~cm} / \mathrm{sec}$. All of these dimensions were doubled for the frame-disk stimuli presented at the far distance so that the stimuli subtended the same visual angles at the two distances, as is indicated in Figure 1. The frames and disks had a luminance of .22 and $1.4 \mathrm{fL}$, respectively, and were presented in an otherwise totally dark visual field. In addition to presenting the two frame-disk stimuli simultaneously, either the right or left frame-disk stimulus at the near or far distance was presented singly. The latter presentations provided control conditions to evaluate the effect of an induced motion of a disk in one frame by the addition of another frame of opposite phase of motion.

All stimuli were viewed binocularly from a dark observation booth. An indicator arrow outside the booth, visible only to the experimenter, signaled the direction of motion of each frame at any instant. This information, together with the observer's response, informed the experimenter whether the induced motion in the disk was that expected from the physical motion of the enclosing frame.

\section{Procedure}

The observers were 25 women and 7 men from a class in general psychology. All had an acuity near and far of at least $20 / 20$, a stereoacuity of at least $25.25 \mathrm{sec}$ of arc, as measured with a Keystone orthoscope, and all were naive as to the purpose of the experiment. Prior to entering the observation booth, general instructions were given by means of a small model of the frames and enclosed disks. The observers were informed that they might or might not see right-left motion in a frame or in a disk and that, if such motion was perceived, its direction and extent were to be reported verbally. Then the observer entered the observation booth and was light adapted before beginning the experiment.

Each observer had four trials consisting of (1) the small pair of frames and enclosed disks at a near distance, (2) the large pair of frames and enclosed disks at the far distance, (3) a single small frame and enclosed disk at the near distance, and (4) a single large frame and enclosed disk at the far distance. The order in which the trials were presented was systematically varied between observers. The tasks of the observers and the order in which the tasks were completed were as follows.
(1) Verbal reports were obtained of the occurrence and magnitude (in inches or feet) of the perceived motion of each frame and disk. (2) If the object appeared to move, the observer indicated when the object appeared to be moving in a particular direction by saying left, left, etc. or right, right, etc., according to the instructions. From the latter report and by referring to the indicator light, the experimenter determined whether the phase of the apparent motion of a disk was the same as or opposite to the phase of the physical motion of the frame enclosing that disk. (3) In the situations in which the two frame-disk stimuli were presented simultaneously, in addition to reporting the apparent directions and magnitudes of the motions, the observer reported whether both disks or both frames appeared to be moving simultaneously and, if so, whether these perceived motions were in the same or in opposite directions (same or opposite phase). Verbal reports also were obtained of (4) the apparent depth (if any) between a disk and its enclosed frame, (5) the apparent width of each frame, and (6) the apparent distance of each frame from the observer's eyes.

\section{RESULTS}

The results from the reports of apparent motion are shown in Table 1. A "+" score for a disk in Table 1 indicates that motion was perceived in the disk and that the phase of this apparent motion was consistent with the induced motion of the disk expected from the physical motion of its enclosing frame. A " + " for a frame indicates that motion was perceived in the frame and that the perceived motion was in the same direction (same phase) as the physical motion of the frame. A " 0 " for either a disk or frame indicates that no motion was perceived in that object. As indicated in Table 1, although apparent motion of the disk did not always occur, whenever it did occur it was in the direction (phase) expected from motion induction from the enclosing frame.

The frequency results (not shown in Table 1) from the reports of the relative phase of motions of the disks in the situations in which two frames and disks were presented simultaneously are as follows. With the two frames at the near distance, 13 observers reported that both disks were moving and that the motions were in opposite directions. Four reported that only one of the two disks was moving and 15 reported no relative

Table 1

Obtained Magnitude (Converted to Centimeters) and Obtained Frequency of Apparent Motion of the Disks and Frames

\begin{tabular}{|c|c|c|c|c|c|c|}
\hline & \multirow{2}{*}{\multicolumn{2}{|c|}{ One Frame and Disk }} & \multicolumn{4}{|c|}{ Two Frames and Disks } \\
\hline & & & \multicolumn{2}{|c|}{ Disks } & \multicolumn{2}{|c|}{ Frames } \\
\hline & Disks & Frames & Left & Right & Left & Right \\
\hline & \multicolumn{6}{|c|}{ Near Distance } \\
\hline Mean & 4.6 & 5.8 & 3.9 & 3.7 & 12.9 & 12.9 \\
\hline Median & 2.5 & 2.5 & 0.0 & 0.0 & 5.1 & 5.1 \\
\hline$\sigma$ & 5.6 & 11.5 & 15.8 & 15.8 & 27.3 & 27.3 \\
\hline Number of + & 30 & 27 & 11 & 8 & 32 & 32 \\
\hline \multirow[t]{2}{*}{ Number of 0} & 2 & 5 & 21 & 24 & 0 & 0 \\
\hline & \multicolumn{6}{|c|}{ Far Distance } \\
\hline Mean & 8.0 & 9.6 & 3.2 & 4.8 & 15.0 & 15.0 \\
\hline Median & 5.1 & 3.8 & 0.0 & 0.3 & 7.6 & 8.9 \\
\hline$\sigma$ & 15.9 & 26.3 & 8.0 & 15.8 & 27.2 & 26.4 \\
\hline Number of + & 30 & 29 & 15 & 16 & 32 & 32 \\
\hline Number of 0 & 2 & 3 & 17 & 16 & 0 & 0 \\
\hline
\end{tabular}


motion of the two disks. With the two frames at the far distance, 20 observers reported simultaneous motion of the two disks in opposite directions, 3 reported only one disk as moving, and 9 reported neither disk as moving. For all observers, at both the near and far distances, the two frames were reported as moving simultaneously in opposite directions.

The average reported width and distance of the frames (converted to centimeters) was 23.6 and $99.6 \mathrm{~cm}$ for the near frames and 30.2 and $124.8 \mathrm{~cm}$ for the far frames. The average report of the distance of a disk relative to its enclosing frame was that the disk appeared to be $3.6 \mathrm{~cm}$ behind the frame at the near distance and $3.3 \mathrm{~cm}$ behind the frame at the far distance. It is of interest to note in Table 1 that there seemed to be some tendency for induced motion to be perceived more frequently when the two frame-disk stimuli were at the far as compared with the near distance. This is consistent with other data that suggest tentatively that there is some tendency for induction effects to increase with increasing distance from the observer even though the size of the stimuli on the eye is unchanged (Gogel, 1975; Gogel \& Newton, 1975).

\section{DISCUSSION}

From the results of Table 1, when two frames and disks were presented simultaneously, the amount of motion induced in a disk by its enclosing frame was reduced as compared with that obtained from the single frame and disk at the corresponding distance. However, even though the two simultaneously presented frames moved in opposite phase, a substantial number of observers saw induced motion in at least one of the two disks, particularly at the far distance. The number of observers (not shown in Table 1) who reported induced motion in both disks when reporting on the apparent motion of each disk separately was 7 for the near and 15 for the far stimulus. In addition, as noted previously, when asked directly about the relative motion of the two disks, $41 \%$ of the observers with the near stimuli and $62 \%$ of those with the far stimuli reported induced motion of the two disks simultaneously in opposite directions. It seems that opposite induced linear motion can occur simultaneously in different parts of the visual field.

The simultaneously induced motion in opposite directions obtained in the present experiment would not necessarily occur if the two frames had been closely adjacent in the visual field. This is consistent with the results obtained by Brosgole (1966) in an experiment in which two frames, each enclosing a stationary luminous sphere, were aligned one above the other, with the frames moving in opposite phase. Twelve of the 24 observers in that study saw the frames as moving and the spheres as stationary. The remaining 12 saw only one of the frames as stationary, with the spheres appearing to move in unison. Also, it will be noted in Table 1 that the perceived motion of a frame in the two frame-disk presentations was considerably greater than the perceived motion of the frames obtained with the single frame-disk stimulus. These considerations suggest the following explanation for the opposite induced motions in the disks that sometimes occurred when the two frames and disks were presented simultaneously. When only one frame and disk were present, much of the motion of the frame was not perceived, with this error contributing to the perception of the motion of the enclosed disk. The presence of a second frame, by providing a motion cue between the frames, increased the tendency to see the frames as moving, thereby reducing the induced motion of the disks. However, since the two frames were separated in direction, the motion cue between the frames was not totally effective, with the result that some induced motion occurred, with the disks appearing to move in opposite directions. The present study indicates that the Roelofs effect cannot explain all instances of induced linear motion. It does not indicate that the Roelofs phenomenon does not contribute importantly to induced motion in more usual cases where only one frame and disk are present, with both of the objects in the same frontoparallel plane. It is hypothesized that an under-perception of the motion of the frame contributes to the perception of motion in the physically stationary disk. From this point of view, any factor, including the Roelofs effect, that increases the perceptual underestimation of the physical motion of the frame is likely to increase the magnitude of the induced motion in the disk.

\section{REFERENCES}

Brosgole, L. An analysis of induced motion (Tech. Rep. NAVTRADEVCEN LH-48). Port Washington, N.Y: U.S. Naval Training Device Center, 1966.

DunCKer, K. Über induzierte Bewegung (Ein Beitrag zur Theorie Optisch Wahregenommerner Bewegung). Psychologische Forschung, 1929. 12, 180-259. (Translated and condensed in W. Ellis, Source book of gestalt psychology. Humanities Press, 1950, Selection 12.)

Gogel. W. C. Depth adjacency and the Ponzo illusion. Perception \& Psychophysics, 1975, 17, 125-132.

Gogel. W. C.. \& Koslow, M. The effect of perceived distance on induced movement. Perception \& Psychophysics, 1971, 10, 142-146.

Gogel, W. C., \& Koslow, M. The adjacency principle and induced movement. Perception \& Psychophysics, 1972. 11. 309-324.

Gogel. W. C., \& Newton, R. E. Depth adjacency and the rod-and-frame illusion. Perception \& Psychophysics, 1975. 18. 163-171.

Roelofs. C. O. Optische Lokalisation. Archiv für Augenheilkunde, 1935, 109, 395-415.

Sugarman, R. C., \& Cohen, W. Perceived target displacement as a function of field movement and asymmetry. Perception \& Psychophysics, 1968, 3, 169-173.

(Received for publication July $18,1977$. ) 